\title{
Long-term impact of maternal high-fat diet on offspring cardiac health: role of micro- RNA biogenesis
}

\author{
Benazir Siddeek', Claire Mauduit², Hassib Chehade', Guillaume Blin', Marjorie Liand', Mariapia Chindamo',
} Mohamed Benahmed ${ }^{2}$ and Umberto Simeoni ${ }^{1}$

\begin{abstract}
Heart failure is a worldwide leading cause of death. Diet and obesity are particularly of high concern in heart disease etiology. Gravely, altered nutrition during developmental windows of vulnerability can have long-term impact on heart health; however, the underlying mechanisms are poorly understood. In the understanding of the initiation of chronic diseases related to developmental exposure to environmental challenges, deregulations in epigenetic mechanisms including micro-RNAs have been proposed as key events. In this context, we aimed at delineating the role of micro-RNAs in the programming of cardiac alterations induced by early developmental exposure to nutritional imbalance. To reach our aim, we developed a human relevant model of developmental exposure to nutritional imbalance by maternally exposing rat to high-fat diet during gestation and lactation. In this model, offspring exposed to maternal high-fat diet developed cardiac hypertrophy and increased extracellular matrix depot compared to those exposed to chow diet. Microarray approach performed on cardiac tissue allowed the identification of a micro-RNA subset which was down-regulated in high-fat diet-exposed animals and which were predicted to regulate transforming growth factor-beta (TGF $\beta$ )-mediated remodeling. As indicated by in vitro approaches and gene expression measurement in the heart of our animals, decrease in DiGeorge critical region 8 (DGCR8) expression, involved in micro-RNA biogenesis, seems to be a critical point in the alterations of the micro-RNA profile and the TGF $\beta$-mediated remodeling induced by maternal exposure to high-fat diet. Finally, increasing DGCR8 activity and/or expression through hemin treatment in vitro revealed its potential in the rescue of the pro-fibrotic phenotype in cardiomyocytes driven by DGCR8 decrease. These findings suggest that cardiac alterations induced by maternal exposure to high-fat diet is related to abnormalities in TGF $\beta$ pathway and associated with down-regulated micro-RNA processing. Our study highlighted DGCR8 as a potential therapeutic target for heart diseases related to early exposure to dietary challenge.
\end{abstract}

\section{Introduction}

A worldwide upward trend in the burden of noncommunicable diseases such as cancers, stroke, heart and

\footnotetext{
Correspondence: Benazir Siddeek (Benazir.Siddeek@chuv.ch)

${ }^{1}$ Woman-Mother-Child Department, Division of Pediatrics, DOHaD Laboratory, Centre Hospitalier Universitaire Vaudois and University of Lausanne, Lausanne, Switzerland

${ }^{2}$ INSERM U1065, Centre Méditerranéen de Médecine Moléculaire (C3M), Team 5, Nice, France

These authors contributed equally: Mohamed Benahmed, Umberto Simeoni Edited by M.V. Nikilson Chirou
}

chronic lung diseases is currently observed. These diseases share common risks factors that are related to lifestyle including smoking, physical inactivity, alcohol and unhealthy diet consumption (World Health Organization (WHO); https://www.who.int/ncds/en/). Importantly, these factors have even more serious impact on health when the exposure occurs during developmental windows of vulnerability such as in utero and early post-natal life. As such, maternal overweight and obesity, the incidence of which are increasing (WHO; http://www.who.int/en/

\section{(c) The Author(s) 2019}

\footnotetext{
(c) Open Access This article is licensed under a Creative Commons Attribution 4.0 International License, which permits use, sharing, adaptation, distribution and reproduction cc) in any medium or format, as long as you give appropriate credit to the original author(s) and the source, provide a link to the Creative Commons license, and indicate if changes were made. The images or other third party material in this article are included in the article's Creative Commons license, unless indicated otherwise in a credit line to the material. If material is not included in the article's Creative Commons license and your intended use is not permitted by statutory regulation or exceeds the permitted use, you will need to obtain permission directly from the copyright holder. To view a copy of this license, visit http://creativecommons.org/licenses/by/4.0/.
} 
news-room/fact-sheets/detail/obesity-and-overweight) and which are often attributed to excessive intake of calorie-dense food, in particular high-fat diets, and reduced physical activity ${ }^{1}$, not only have consequences in the short term with pregnancy complications, but also impact offspring health later during life. Maternal obesity and dietary imbalances are particularly of high concern in the early origins of heart failure ${ }^{2,3}$. Since human cardiomyocyte have limited proliferative capacity, the exposure of the developing heart to environmental challenges such as nutritional excess may have deleterious effects on lifelong cardiomyocyte functions ${ }^{4}$. Indeed, maternal exposure to high-fat diet and maternal obesity increases the risk for impaired cardiac function in adulthood by modifying heart rate and inducing left ventricular wall thickness, hypertrophy ${ }^{5}$ and fibrosis ${ }^{6,7}$. In the early origins of heart diseases, epigenetic mechanisms are likely key players. In particular, micro-RNAs, which belong to small non-coding RNAs (around 22 nucleotides in length) involved in each step of heart development, seem to play a crucial role in the unfavorable programming of heart diseases $^{8}$. By controlling about $60 \%$ of all protein-coding genes in mammals, micro-RNAs are considered as significant post-transcriptional regulators of gene expression $^{9}$, and regulate almost all biological processes including development, differentiation, proliferation, apoptosis, metabolism and tissue remodeling. The critical role of maintaining micro-RNA balance in the heart has been revealed notably in models of mice where cardiomyopathies were induced by inhibiting the expression of proteins involved in micro-RNA biogenesis in cardiomyocytes ${ }^{10-12}$. Micro-RNA biogenesis is a regulated multistep process starting in the cell nucleus where primary micro-RNAs (pri-micro-RNAs) are transcribed mainly by RNA polymerase II. Through the action of a microprocessor complex including nuclear ribonuclease III (DROSHA) and its cofactor DiGeorge critical region 8 (DGCR8), pri-micro-RNAs are trimmed into 70 nucleotide hairpins called precursor micro-RNAs (pre-microRNAs $)^{13}$. Then, pre-micro-RNAs assembles into a complex constituted by Exportin-5 (XPO5) and RanGTP and translocate into the cytoplasm ${ }^{14}$ where they continue their maturation, through a splicing realized by a complex including DICER and TAR RNA-binding protein 2 (TRBP). This results in RNA duplexes comprising the mature micro-RNAs and the passenger strand, microRNAs $^{* 15}$. Finally, each mature single strand micro-RNA strand is incorporated into the RNA-induced silencing complex (RISC) comprising an Argonaute (AGO) protein to direct the silencing of the targeted $\mathrm{mRNA}^{16}$. Interestingly, the micro-RNA biogenesis machinery is sensitive to hormonal regulation and dietary changes ${ }^{17-19}$. In this context, micro-RNAs and their regulation represent a potential candidate in the understanding of the long-term effects on cardiac functions induced by exposure to nutritional challenges early during development. Thus, based on a human relevant model of cardiac alterations programmed by exposure to maternal high-fat diet, we aimed at delineating the role of micro-RNAs, identifying the defects in the micro-RNA biogenesis machinery in such programming and at highlighting potential targets and approaches for the phenotype reversal.

\section{Results}

Exposure to maternal high-fat diet induces cardiac hypertrophy and increases cardiac extracellular matrix depot

At post-natal day 77 (PND77), whereas no difference was detected in the body weight of animals exposed to chow diet (CTRL) or to high-fat diet (HFD) (supplementary data S1), increased heart vs. body weight ratio was induced by maternal exposure to high-fat diet (Fig. 1a). This was associated with enlarged cardiomyocytes visualized by hematoxylin-eosin staining (Fig. 1b). In addition, exposure to high-fat diet increased extracellular matrix depot as indicated by Masson's trichrome staining (Fig. 1c). These data indicate that maternal exposure to high-fat diet induced cardiac remodeling with hypertrophic and fibrotic patterns, without changes in the body weight at PND77.

\section{Exposure to maternal high-fat diet alters adult cardiac micro-RNA levels}

Since micro-RNAs have been described as effectors of environmental influences on gene expression and disease $^{20}$, and because alterations in micro-RNA balance have been reported to induce cardiac hypertrophy and fibrosis, we wondered what could be the role of microRNAs in the cardiac tissular alterations induced by maternal exposure to high-fat diet. With such an aim, we analyzed the micro-RNA profile by microarray experiment. The microarray quality control data are presented in supplemental data S2. The comparison of micro-RNA profile between rats exposed to chow diet (CTRL) or to HFD highlighted 19 down-regulated micro-RNAs, with an adjusted $P$ value $<0.05$ (Fig. 2a). Nine micro-RNAs (let-7g, miR-15, miR-21, miR-27a, miR-29c, miR-33, miR-101a, miR-218a and miR-450a) were selected for quantitative real-time PCR (RT-qPCR) validation of the microarray data (Fig. 2b). Except for miR-15 where no modification was detected, RT-qPCR data correlated well with those obtained from the microarray for all selected micro-RNAs, confirming the down-regulation of microRNA levels in the heart of animals exposed to maternal high-fat diet compared to the ones exposed to chow diet. All the 19 significantly differentially expressed 


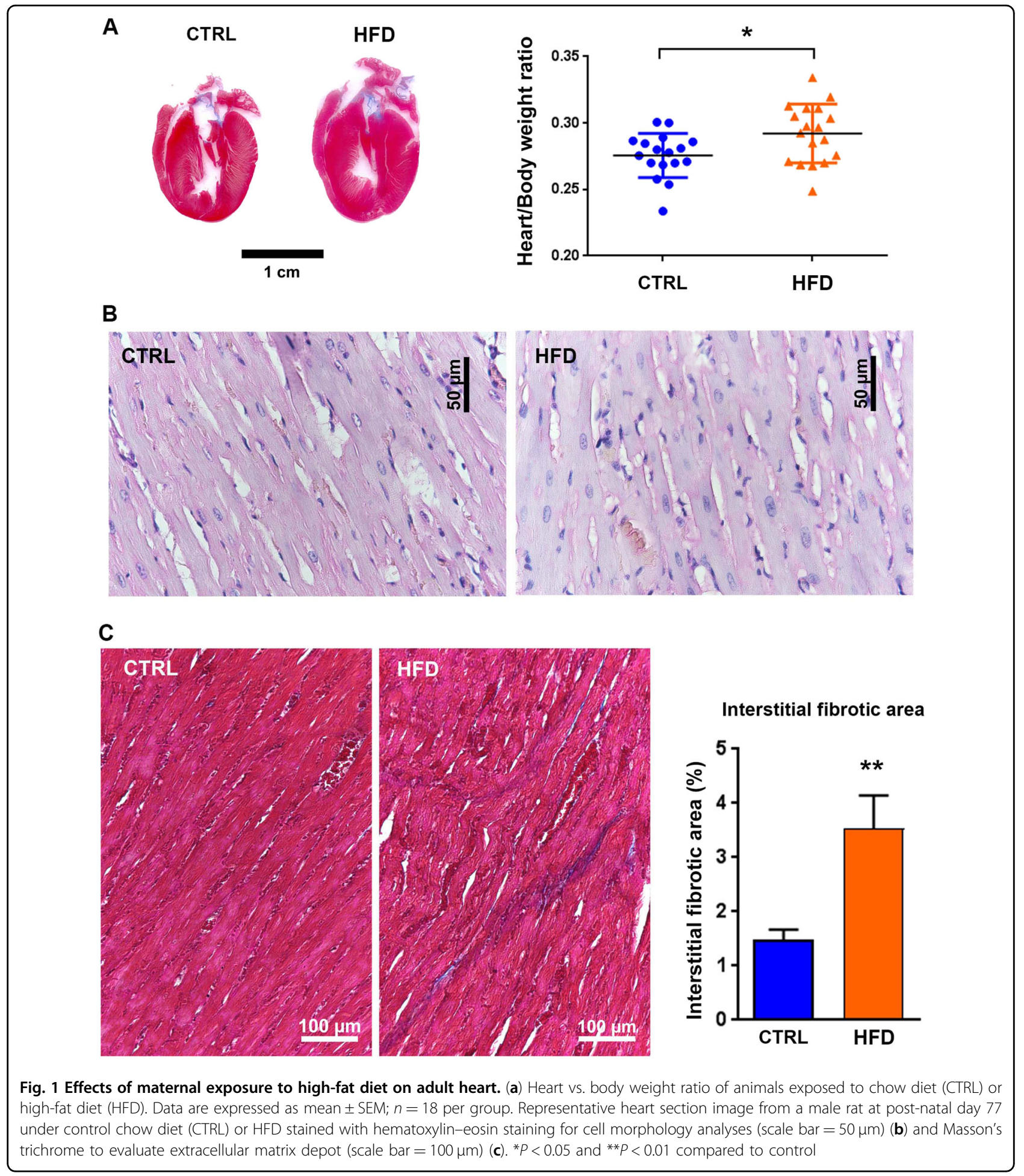

micro-RNAs have previously been reported in experimental or human cardiovascular diseases (supplemental table 2). The targets of the altered micro-RNAs have been reported to play a role in metabolism, cardiogenesis, cell survival, hypertrophy and fibrosis (Fig. 3). Collectively, these data suggest that tissular cardiac alterations observed in the heart of animals exposed to maternal high-fat diet are linked to the down-regulation of a microRNA subset regulating cellular mechanisms that are critical for cardiac functions. 


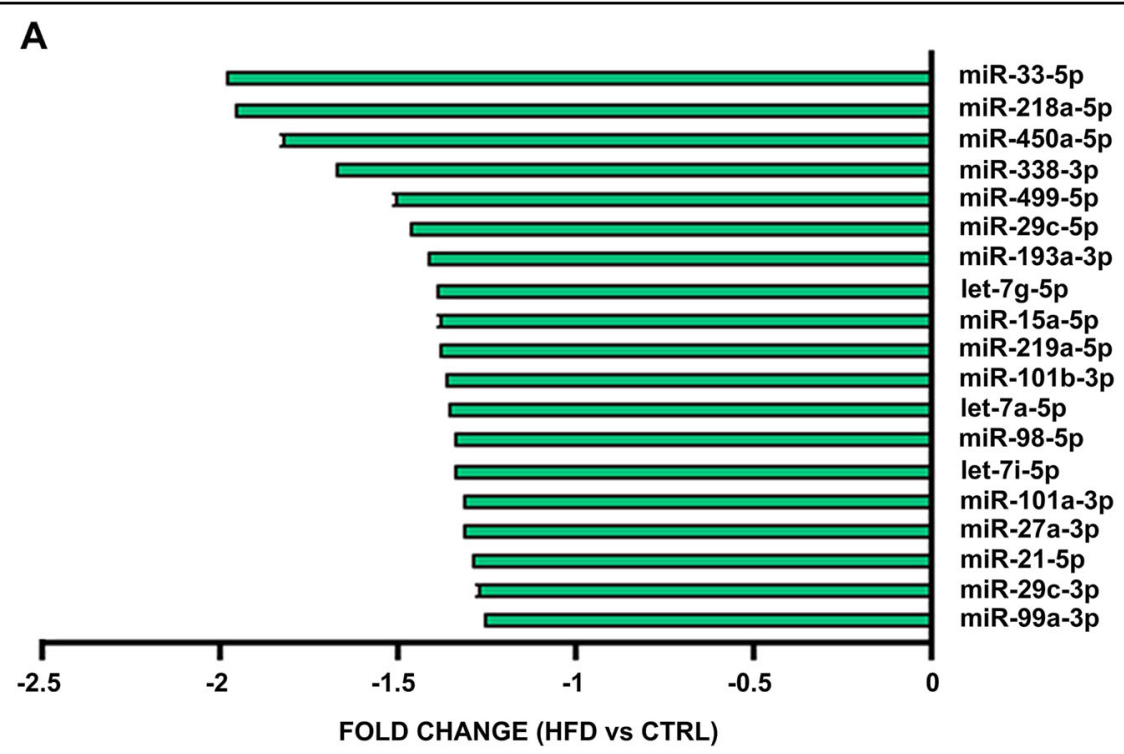

B

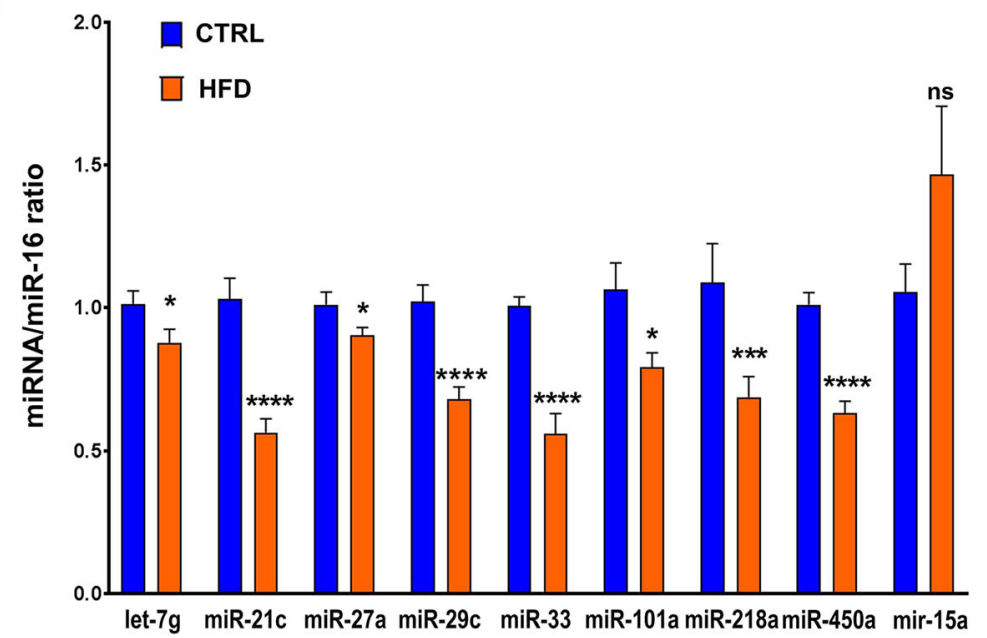

Fig. 2 Effects of maternal exposure to high-fat diet on adult cardiac micro-RNA levels. (a) Differential expression of micro-RNAs detected by microarray in the heart of male rats at post-natal day 77 exposed to maternal high-fat diet compared to chow diet. (b) let-7g, miR-15, miR-21, miR-27a, miR-29c, miR-33, miR-101a, miR-218a and miR-450a levels were analyzed by quantitative real-time PCR (RT-qPCR) in the heart of male rats at post-natal day 77 under control chow diet (CTRL) or high-fat diet (HFD). Data are expressed as mean $\pm S E M ; n=13$ per group. ${ }^{*} P<0.05$, ${ }^{* * *} P<0.001$ and ${ }^{* * * *} P<0.0001$ compared to control, ns not statistically significant

\section{Altered cardiac micro-RNAs are involved in cardiac diseases and associated pathways}

To identify regulatory networks involved in the adult heart dysfunctions induced by maternal exposure to highfat diet, we performed a computational target prediction analysis coupled to pathway analysis using DIANAmiRPath (v3.0) software. Notably, KEGG (Kyoto Encyclopedia of Genes and Genomes) pathway analysis showed that the altered micro-RNAs are predicted to target extracellular matrix-receptor interaction, fatty acid degradation and metabolism. The DIANA-miRPath software calculates the significance for all the miRNA-mRNA pairs in a pathway, and then combines them into a merged $P$ value for each pathway ${ }^{21}$. The results are reported as heat maps, and the pathways are clustered based on significance levels (Fig. 4a). A closer look at the altered micro-RNA targets revealed that the majority of the altered micro-RNAs are involved in transforming growth factor-beta (TGF $\beta$ ) signaling which is closely linked to extracellular matrix-receptor interaction and involved in cardiac fibrosis and hypertrophy (Fig. 4b). In the response to cardiac stress, TGF $\beta$ is subsequently activated and triggers a cascade of signaling involving SMAD protein phosphorylation (canonical pathway) or the activation of other intermediates such as mechanistic target of rapamycin (mTOR) or mitogen-activated protein 


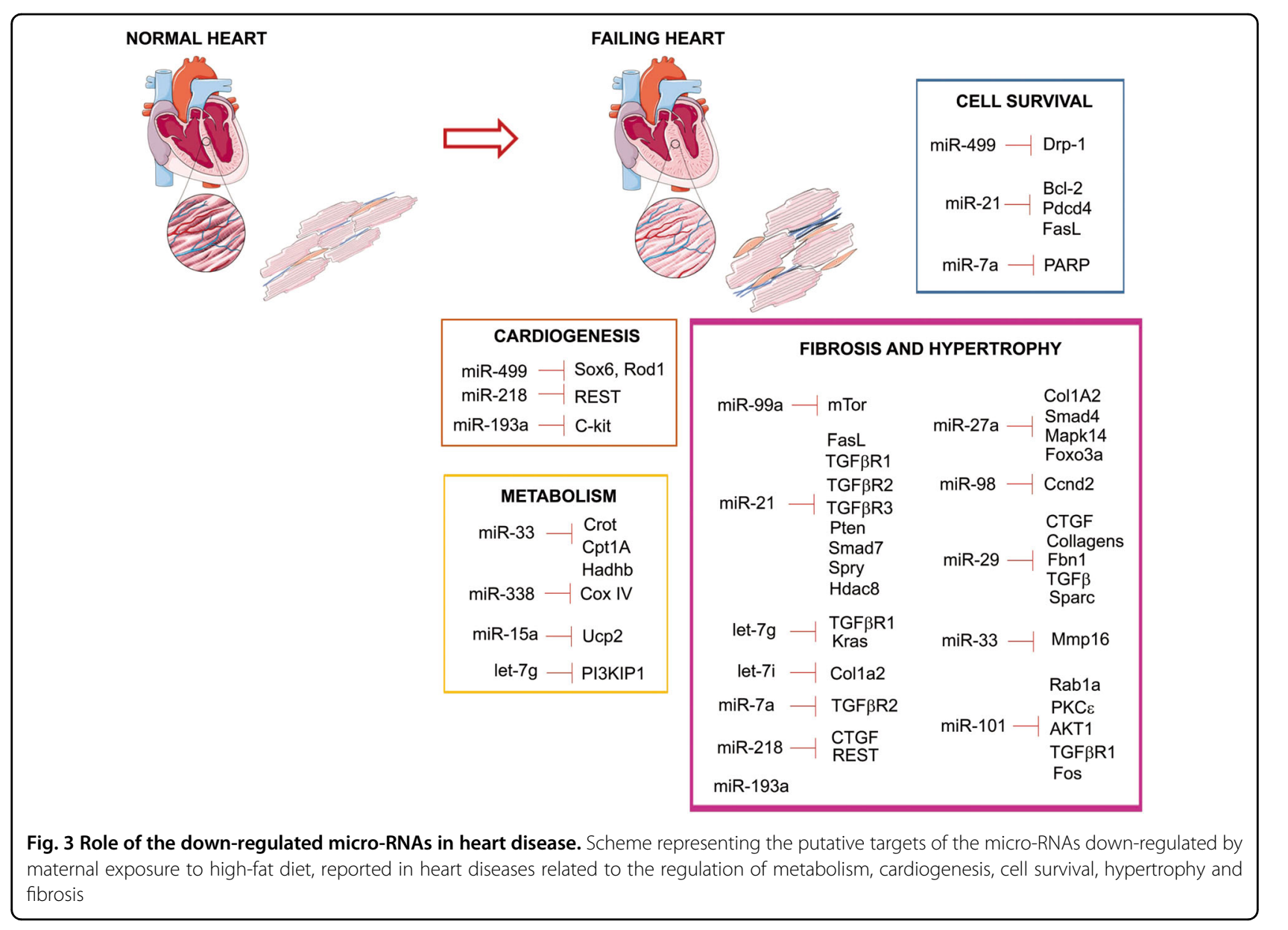

kinase (MAPK) (non-canonical pathway), leading to the up-regulation of the expression of genes involved notably in the regulation of extracellular matrix synthesis and cell growth (Fig. 4b). As such, the dysregulation of TGF $\beta$ pathway has been shown to result in fibrosis and hypertrophy $^{22}$. In light of these points, we supposed that the cardiac hypertrophy and fibrosis induced by maternal exposure to high-fat diet are due to micro-RNA-mediated TGF $\beta$ pathway dysregulation.

Exposure to maternal high-fat diet alters the expression of genes involved in TGF $\beta$ regulated hypertrophy and fibrosis

To verify whether the micro-RNA down-regulation was associated with alterations in the TGF $\beta$ pathway in the heart of animals exposed to maternal high-fat diet, we measured the expression levels of the predicted targets of these micro-RNAs, involved in TGF $\beta$-mediated fibrosis and hypertrophy. Effectively, in the heart of animals exposed to high-fat diet compared to chow diet, increased transforming growth factor-beta receptor type III (TGFßR3) mRNA levels were detected (Fig. 5a). This was associated with increased expression of pro-hypertrophic genes such as V-Ki-ras2 Kirsten rat sarcoma viral oncogene homolog (K-RAS), protein kinase C epsilon (PRKCE), increased phosphorylation of ribosomal protein s6 kinase (RPS6KB1, a target of mTOR), and increased expression of pro-fibrotic genes such as connective tissue growth factor (CTGF) and secreted protein acidic cysteine-rich (SPARC) (Fig. 5b). These data suggest that cardiac hypertrophy and fibrosis induced by maternal exposure to high-fat diet might be due to micro-RNAmediated TGF $\beta$ pathway dysregulation at multiple levels.

\section{Maternal exposure to high-fat diet modifies the expression of proteins involved in micro-RNA processing}

Prompting a search for underlying mechanisms in micro-RNA down-regulation induced by maternal exposure to high-fat diet, we investigated the different steps of micro-RNA biogenesis by measuring the expression levels of key components of the micro-RNA biogenesis machinery: DROSHA, DGCR8, DICER, XPO5 and AGO2 (Fig. 6a). Among them, DGCR8 showed a significant decrease at the protein (Fig. 6a) and mRNA (Fig. 6b) levels when the animals were exposed to maternal high-fat diet. These data suggest that DGCR8-decreased expression may be responsible for micro-RNA down-regulation 


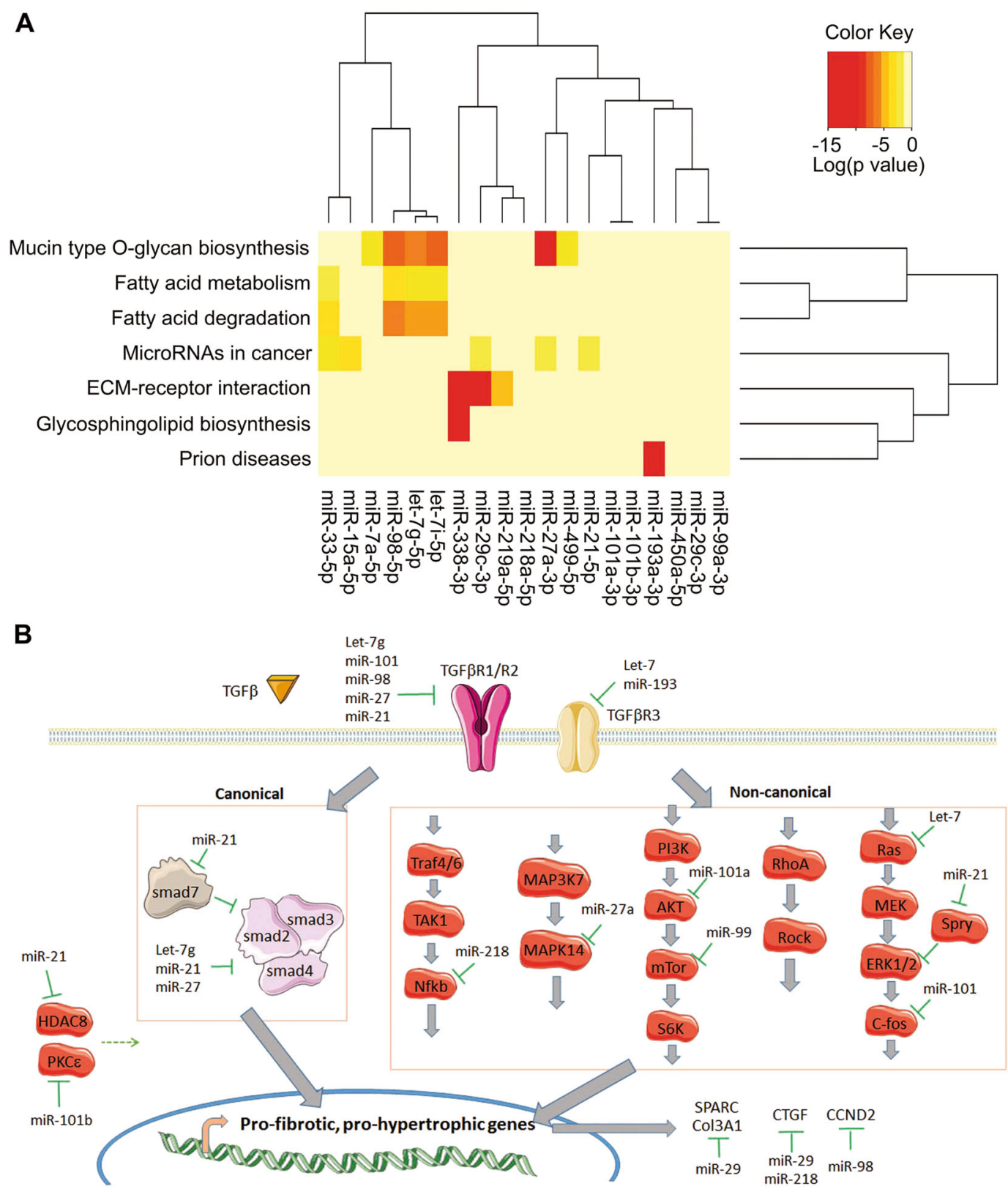

Fig. 4 Effects of maternal exposure to high-fat diet on the expression of genes involved in transforming growth factor-beta (TGF $\beta$ )regulated hypertrophy and fibrosis. (a) Cluster heat maps showing the pathways which are predicted to be altered based on significance levels. The more intense red color indicates higher probability that a specific pathway is significantly enriched with target genes for a certain micro-RNA. (b) Scheme representing the involvement of down-regulated micro-RNAs in the regulation of canonical and non-canonical TGF $\beta$ pathways. Putative inhibition of genes by micro-RNAs is represented by green line. Dotted lines represent direct or indirect stimulation of the pathway

induced by maternal exposure to high-fat diet, and the subsequent cardiac alterations mediated by TGF $\beta$ pathway dysregulations.

\section{DGCR8 down-regulation effects on micro-RNA levels and TGF $\beta$ pathway}

To delineate the role of DGCR8 in the regulation of micro-RNA levels in cardiomyocytes, we down-regulated its expression in $\mathrm{H} 9 \mathrm{C} 2$ cell line. In these cells, the transfection of a specific gapmeR targeting $\operatorname{Dgcr} 8$ (gap DGCR8) induced a $74 \%$ decrease in $D g c r 8$ mRNA levels compared to the cells transfected with a control gapmeR (negative control, gap CTRL) (Fig. 7a). Difference in cell survival was not detected when Dgcr8 was inhibited (supplemental data). In contrast, the levels in let-7g, miR-21, miR-27a, miR-29c, miR-33, miR-101a and miR-218a were 


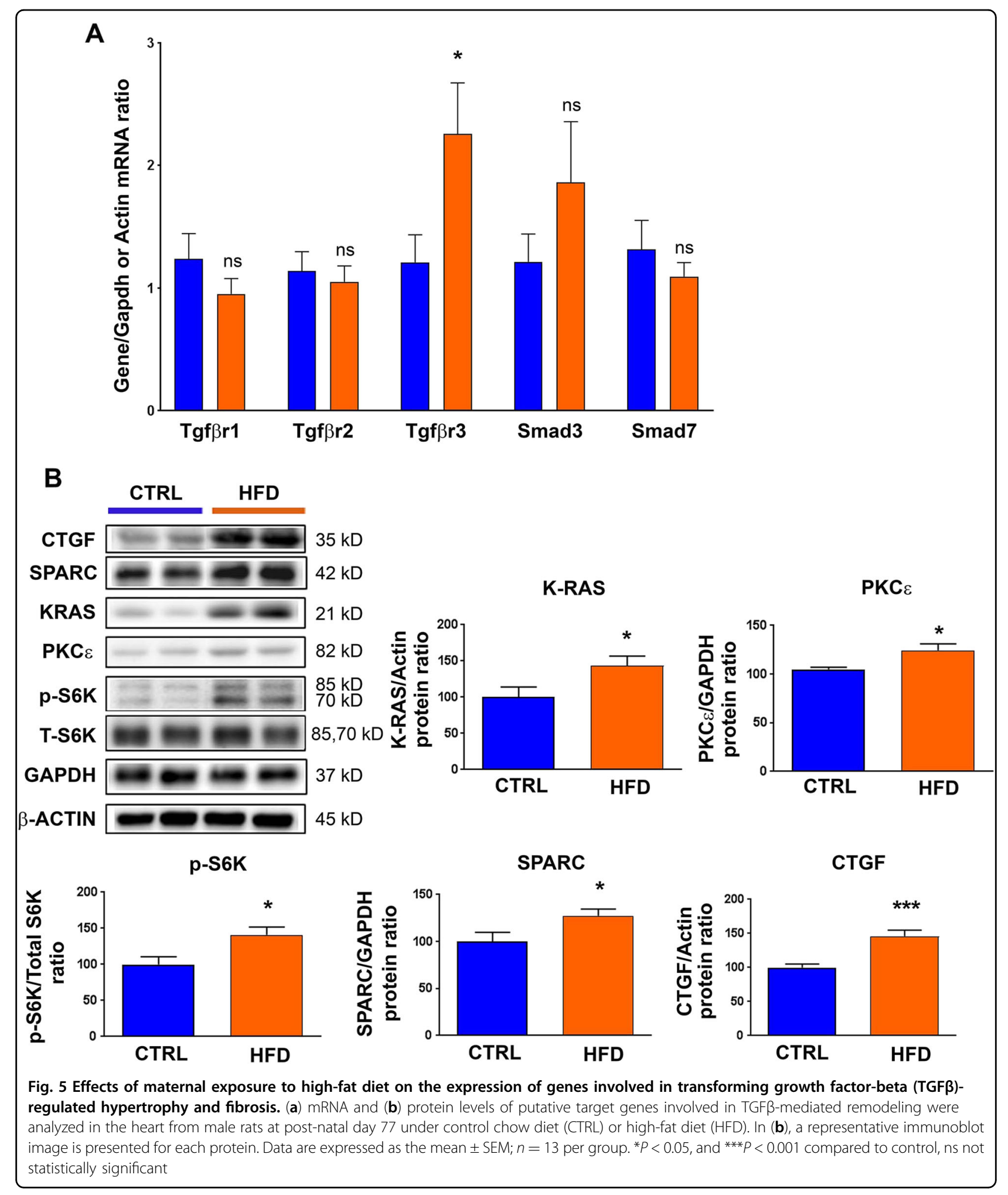

down-regulated in these cells (Fig. 7b). We then verified the role of $D g c r 8$ and micro-RNA decrease on the regulation of genes involved in TGF $\beta$-mediated fibrosis and hypertrophy pathway (Fig. 7b, c). We found that Dgcr8 down-regulation through gapmeR transfection induced a significant increase in Tgfßr3 (Fig. 7b). The mRNA levels 


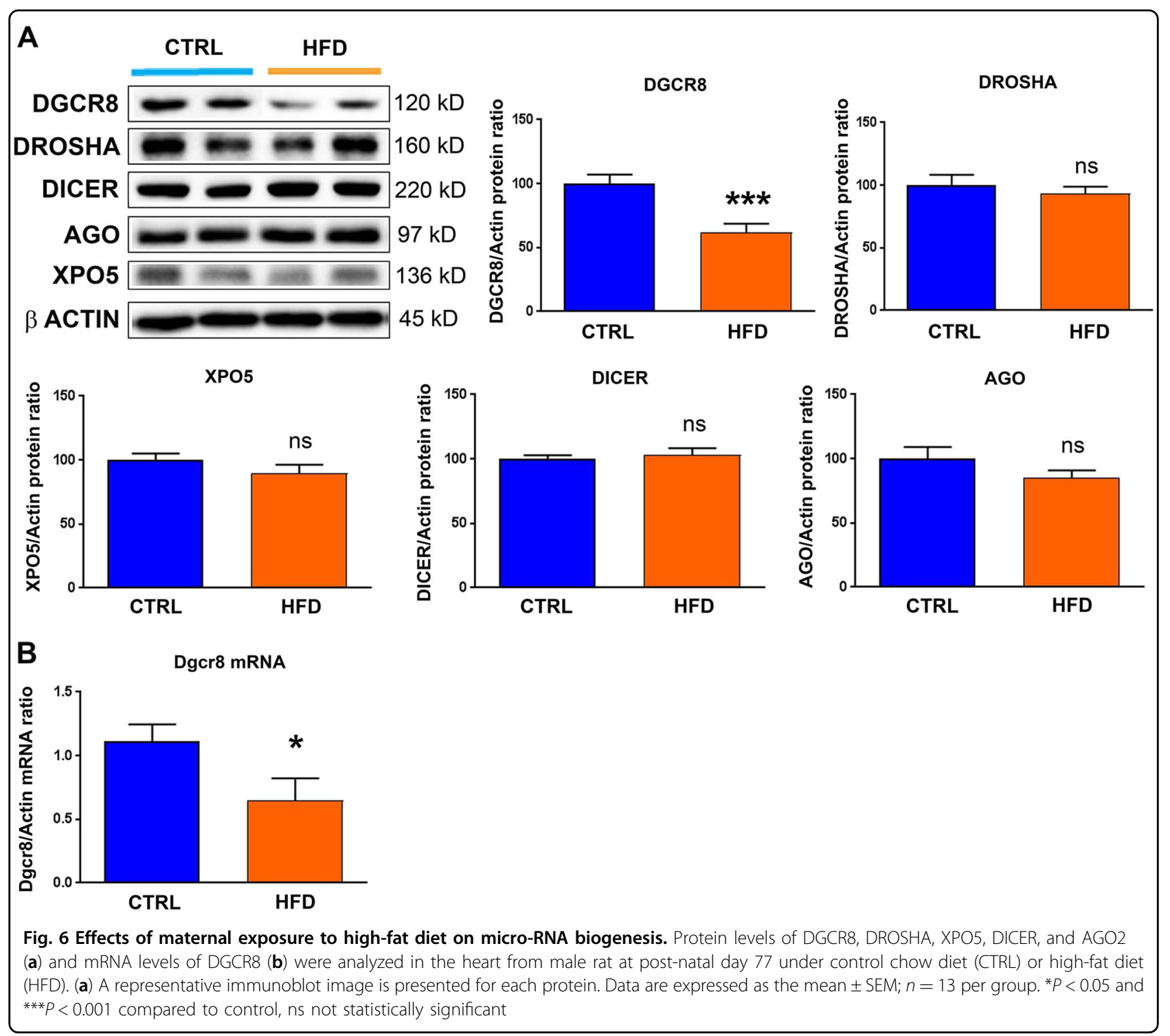

of pro-hypertrophic genes such as K-Ras, mTor, Hdac8, Prkce and $C c n d 2$ were upregulated when $D g c r 8$ expression was decreased (Fig. 7c). Similarly, pro-fibrotic genes such as Ctgf, Sparc, Col3a1 and FasL showed increased mRNA levels in cells where Dgcr8 expression was inhibited (Fig. $7 \mathrm{~d}$ ). These data indicate that $D g c r 8$ decrease in vitro can induce the down-regulation of a subset of micro-RNAs involved in TGF $\beta$ pathway regulation, through the regulation of multiple targets involved in the canonical and non-canonical pathways. This results in increased pro-hypertrophic and pro-fibrosis gene expression in cardiomyocytes. In view of these results, DGCR8 decrease appears as a potential origin for the cardiac fibrosis and hypertrophy driven by maternal exposure to high-fat diet.
Hemin treatment rescues the levels of micro-RNAs and genes in cardiomyocytes with low levels of $D g c r 8$

Since DGCR8 down-regulation leads to alterations in cardiomyocytes, we wondered if we could rescue the phenotype by increasing DGCR8 expression and/or activity. Thus, we further tested the rescue of the phenotype through hemin treatment in cardiomyocytes where Dgcr8 expression was decreased. Hemin, which is a ferric form $(\mathrm{Fe} 3+)$ of heme and which has been shown to stimulate the microprocessor activity in vitro ${ }^{23-25}$ and in vivo ${ }^{26}$, has been described as an efficient approach in the reversal of fibrosis in a model of hypertension ${ }^{27}$, and as a negative regulator of TGF $\beta$ pathway ${ }^{28}$. Therefore, we wondered if these beneficial effects of hemin on fibrosis and TGF $\beta$ pathway described in other models could be 


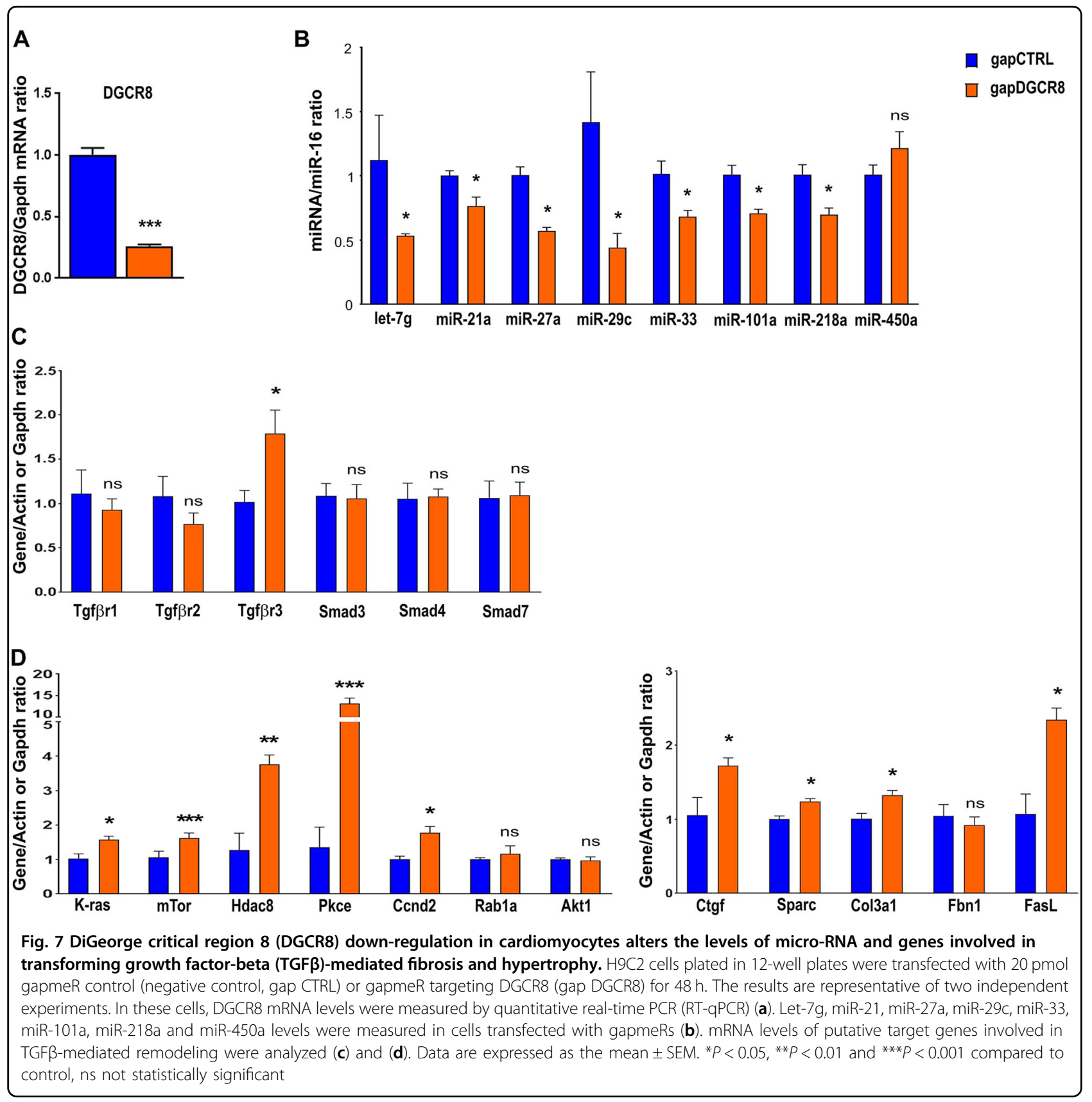

also found in cardiomyocytes, and if those effects are mediated by DGCR8 and micro-RNAs. To answer this question, cardiomyocytes were treated with $1 \mu \mathrm{M}$ hemin. These cells exhibited increased $D g c r 8$ mRNA levels compared to control cells (treated with vehicle) (Fig. 8a). Furthermore, in cells transfected with gapmeRs, the down-regulation in the anti-fibrotic micro-RNA miR101a and let-7g levels induced by $D g c r 8$ inhibition was reduced when the cells were treated with hemin (gap DGCR8 with hemin vs. gap CTRL) (Fig. 8b). Finally, the increase in pro-fibrotic genes induced by $\operatorname{Dgcr} 8$ inhibition, including Tgf $\beta r 3$, Ctgf and Col3a1, was not detected anymore when the cells were treated with hemin (gap DGCR8 with hemin vs. gap CTRL) (Fig. 8c). Overall, these data suggest that increasing DGCR8 expression and/or activity in cardiomyocytes with hemin tends to rescue the pro-fibrotic phenotype mediated by TGF $\beta$ pathway.

\section{Discussion}

Our study addresses critical health problems (obesity and heart diseases) associated with modern lifestyle diet (high-fat diet). Maternal obesity and high-fat diet have been associated with increased risk for heart diseases, notably through the induction of cardiac remodeling. 


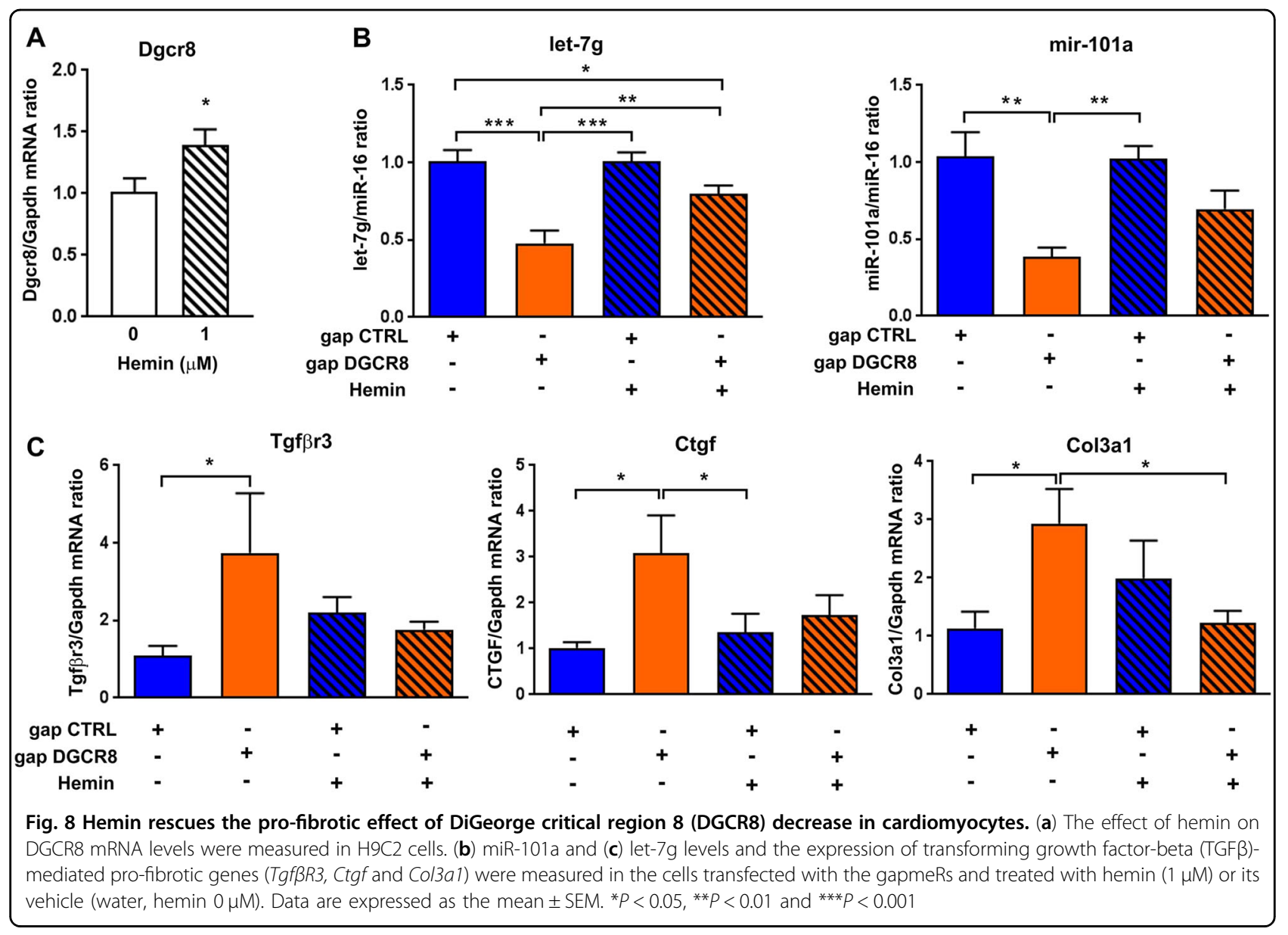

Indeed, maternal exposure to high-fat diet induces cardiac hypertrophy and fibrosis that are observed early during development (at PND1 and PND10),29. Our current study showed that maternal exposure to high-fat diet induced long-term histological and molecular changes in the heart prior to the onset of overt obesity. Notably, the present work highlighted the decrease in a micro-RNA subset as a potential mechanistic basis linking maternal nutritional imbalance early during development and mortality related to cardiac diseases. Here, the downregulation of a subset of cardiac micro-RNAs induced by maternal exposure to high-fat diet for 35 days, during pregnancy and lactation, was detected later during life, in adulthood. Importantly, this observation indicates that a transient exposure to dietary distress during developmental sensitive periods induces sustained downregulation of a micro-RNA subset. This observation is reminiscent of the professor Barker hypothesis related to the concept of the developmental origins of health and disease. In this context, it is noteworthy that altered micro-RNA patterns may be associated with disturbed developmental processes during fetal or neonatal life. Here, our work further highlighted micro-RNA alterations as a possible cause for TGF $\beta$-mediated cardiac fibrosis and hypertrophy induced by early developmental exposure to dietary challenge. Various intracellular signaling pathways are thought to play a critical role in the pathological remodeling of the heart and, importantly, are activated both independently and downstream of TGF- $\beta$. Among the targets we identified in this work, Tgf $\beta r 3$ was up-regulated. TGFBRIII is a widely expressed accessory receptor $^{30}$ for multiple classes of growth factors (including TGF- $\beta$ s, along with fibroblast growth factor (FGF), inhibin, activin, bone morphogenetic protein (BMP)-2, BMP-4 and BMP-7), and as such, TGF $\beta$ RIII has the potential to influence diverse cellular processes. TGF $\beta$ RIII enhances TGF $\beta$ signaling ${ }^{31,32}$ and also activates RAS-ERK and p38 MAPK in a ligand-independent manner, both involved in fibrosis ${ }^{33}$ and hypertrophy, in a SMADindependent manner. Importantly, we newly identified alterations in DGCR8 expression as a potential cause for long-term cardiac micro-RNA down-regulation in this model. DGCR8 importance in micro-RNA biogenesis and in pathophysiology has been revealed in human 22q11.2 deletion syndrome (known as DiGeorge syndrome $)^{34}$ and in animal model where the Dgcr8 gene was 
heterozygously deleted ${ }^{35}$. The haploinsufficiency of Dgcr8 reduces the abundance of DGCR8 protein, resulting in lower pri-micro-RNA processing efficiency and abnormal expression of a subset of micro-RNAs ${ }^{36}$. In humans, the DiGeorge syndrome is associated with increased risk for congenital heart defects ${ }^{37}$. In mice, cardiomyocytespecific deletion of $D g c r 8$ induces cardiac fibrosis with left ventricular malfunction progressing to a dilated cardiomyopathy and premature lethality ${ }^{12}$. Our data highlighted a new potential pathway through which DGCR8 alterations could lead to cardiac hypertrophy and fibrosis involving at least in part TGF $\beta$ pathway. Although modulation of TGF- $\beta$ signaling with different approaches has proven effective pre-clinically and clinically in the treatment of multiple pathologies ${ }^{38-40}$, the rescue of the phenotype remains partial. In the light of our findings, DGCR8 appears as an attractive candidate in the reversal of the cardiac phenotype induced by early exposure to dietary challenge since its modulation might impact the TGF $\beta$ signaling pathway at multiple levels. In this sense, studies have demonstrated the possibility of regulating DGCR8 expression and activity in several ways including by cofactor binding, phosphorylation, acetylation and proteolytic cleavage ${ }^{26,41-43}$. Among them, hemin, a ferric form (Fe3+) of heme, has proven its efficiency in the upregulation of DGCR8 activity. Hemin is incorporated in a DGCR8 dimer and stimulates the microprocessor activity in vitro ${ }^{23-25}$ and in vivo ${ }^{26}$. Mechanistically, DGCR8 was shown to depend on hemin to recognize the apical loop of primary micro-RNA ${ }^{44}$. Furthermore, the hemin level in human cells can impact the expression of several micro$\mathrm{RNAs}^{26}$ and decrease cardiac oxidative stress and fibrosis in a rat model of systemic hypertension ${ }^{27}$. Here, we showed that hemin can restore micro-RNA levels in cardiomyocytes while $\operatorname{Dgcr} 8$ expression was decreased, supporting the physiological function of hemin in stimulating micro-RNA biogenesis and benefits for heart functions. Similarly, through the stable binding to DGCR8, Cobalt (III) protoporphyrin has been shown to compensate micro-RNA processing deficiency and restore micro-RNA levels in models where DGCR8 expression was decreased in vivo ${ }^{45}$. Thus, it is of high interest to test the potential of hemin treatment in the prevention and/or reversal of cardiac alterations in animals exposed to dietary challenge early during development.

More widely, alterations in primary micro-RNA processing have been reported in other models of exposure to environmental challenges such as cigarette smoke ${ }^{46}$, and also in aging ${ }^{47}$ and in a number of human diseases including cancer ${ }^{48}$, schizophrenia ${ }^{49}$, kidney ${ }^{50}$ and autoimmune diseases ${ }^{51}$. To restore micro-RNA processing by enhancing the activity of DGCR8 protein using small molecule agents represents an interesting candidate. Thus, the targeting of DGCR8 offers opportunities for intervention and may emerge as an exciting and important arena for drug development. Also, micro-RNAs are strikingly stable and can be detected in body fluids, including blood. This observation has raised the possibility that micro-RNAs may be probed in the circulation and can serve as novel diagnostic markers ${ }^{52,53}$. Thus, this is of high interest to evaluate the potential of the microRNAs identified in our study as early blood-based biomarkers of cardiac alterations induced by exposure to dietary challenges. Our micro-RNA profile could provide a novel strategy for diagnosis in chronic cardiac dysfunctions programmed by environmental stressors early in life.

\section{Methods \\ Materials}

Protease inhibitor cocktail, Tween-20, Hemin, High Pure miRNA Isolation Kit and other reagents were purchased from Sigma Aldrich. Antibodies raised against DGCR8, $\beta$-actin, anti-rabbit and anti-mouse IgG HRPlinked antibody; BCA Protein Assay Kit; RIPA buffer; phosphatase inhibitor cocktail; chemiluminescent western pico plus chemiluminescent substrate, Power up SybrGreen Master Mix, High Capacity Reverse Transcriptions Kit, TaqMan Advanced miRNA cDNA Synthesis Kit and miRNA Advanced Taqman assays were purchased from Thermo Fisher Scientific, Inc. The polyvinylidene difluoride (PVDF) membranes were obtained from Amersham. DICER, DROSHA, XPO5, CTGF, SPARC and GAPDH antibodies were obtained from Santa Cruz Biotechnology. Antibodies raised against phospho-p70 S6K and p70 S6K were purchased from Cell Signaling Technology, Inc. GapmeRs were purchased from Qiagen.

\section{In vivo experimental studies}

The animal experiments were performed in accordance with EU legislation (Directive 2010/63/EU) and approved by a local animal care and use committee (Comité Institutionnel d'Éthique Pour l'Animal de LaboratoireCIEPAL-Azur-Agreement number: NCE-2013-109; Protocol No. 2015-62). Pregnant Sprague Dawley rats at gestational day 7 (GD7) (Janvier, Le Genest Saint Isle, France) were individually housed in temperaturecontrolled rooms with $12 \mathrm{~h}$ light $/ 12 \mathrm{~h}$ dark cycles and ad libitum access to water and feed. Upon arrival, pregnant females were either fed with chow diet (R03, Safe, Augy, France, $n=5$ ) or high-fat diet (SNIFF 60\%, SSNIFF, Soest, Germany, so-called HFD, $n=5$ ). At birth, pups were sexed by anogenital distance and litters were culled to 5 pups per dam by removing or adding female pups as necessary. The remaining pups were killed by $\mathrm{CO}_{2}$ inhalation. At PND21, all male rats were given a chow (R03). Animals were killed by $\mathrm{CO}_{2}$ inhalation on PND77 (young adults). Each group contains 18 male rats. 


\section{Heart sampling}

The heart was quickly harvested and immediately frozen in liquid nitrogen or fixed in paraformaldehyde and paraffin embedded. Frozen tissues were grounded into fine powder for further molecular analyses.

\section{Histology, immunohistochemistry and immunofluorescence}

Heart sections $(5 \mu \mathrm{m})$ were stained with Masson's trichrome. Five animals per group were analyzed. Image acquisition was performed on a Nikon Eclipse Ti microscope. Fibrotic area quantification was performed as described by Chen et al. ${ }^{54}$ with Image J software.

\section{Cell culture and transfection}

H9C2 cardiomyocytes cell line was maintained in medium supplemented with $10 \%$ heat-inactivated fetal bovine serum at $37^{\circ} \mathrm{C}$ in a humidified, $\mathrm{CO}_{2}$-controlled (5\%) incubator. Cells were transfected with 20 pmol gapmeR targeting Dgcr8 (gap DGCR8) or a negative control using Lipofectamine RNAimax Transfection reagent, according to the manufacturer's protocol. At $48 \mathrm{~h}$ following transfection, cells were harvested for RNAs and protein extraction. For rescue experiments, $24 \mathrm{~h}$ after transfection, cells were incubated with $1 \mu \mathrm{M}$ hemin, or with the vehicle (water) for $48 \mathrm{~h}$.

\section{Cell survival assay}

Cells were seeded on 96-well plate and transfected with gapmeRs. Cells were washed with phosphate buffer saline, fixed in ice-cold methanol for $10 \mathrm{~min}$ and stained with $0.5 \%$ crystal violet solution at room temperature for 10 min. Optical density was measured on a plate reader Infinite M200Pro (Nanoquant).

\section{Protein extraction}

Aliquots ( $20 \mathrm{mg})$ of heart tissue powder were homogenized in RIPA buffer (supplemented with 1\% proteases and phosphatases inhibitor cocktail). Protein concentration was determined with the bicinchoninic acid assay. In total, 10 to $30 \mu \mathrm{g}$ were used for western blot analysis.

\section{Western blotting analysis}

Lysates were separated using sodium dodecyl sulfate-polyacrylamide gel electrophoresis (SDS-PAGE) and transferred to PVDF membranes. The membranes were blocked with $1 \%$ bovine serum albumin in phosphate-buffered saline ( $\mathrm{pH}$ 7.4) containing $0.05 \%$ Tween-20 and were incubated with primary antibodies and horseradish peroxidase (HRP)-conjugated secondary anti-rabbit or -mouse antibodies. The proteins of interest were visualized using SuperSignal West Pico PLUS Chemiluminescent Substrate. Membranes were scanned with a luminescent image analyzer camera G:Box (Syngene) and quantified with Gene Tools software (Syngene).

\section{RNA isolation}

Total RNA was isolated from frozen heart powder using $1 \mathrm{ml}$ TRIzol reagent and $200 \mu \mathrm{l}$ chloroform. The aqueous phase was precipitated with $1.5 \mathrm{vol}$ of ethanol $100 \%$ at $4{ }^{\circ} \mathrm{C}$ overnight followed by $75 \%$ ethanol. Total RNAs were further purified on a column. The RNA purity was checked by optical density measurement at 230, 260 and $280 \mathrm{~nm}$. The quantities of total RNAs and micro-RNAs were evaluated with fluorescent-based methods on a Qubit apparatus (Life technologies, Inc.). RNA integrity was evaluated with a Fragment Analyzer (Agilent).

\section{MIRNA microarray experiment}

Sample quality was tested and was ranging from RQN (RNA Quality Number) 6.7 to RQN 8.0.

To assess the effect of maternal exposure to high-fat diet on cardiac miRNA levels, RNAs from four biological replicates of high-fat diet vs. control diet were hybridized on the microarrays (SurePrint Rat miRNA Microarrays, Agilent). Each sample was prepared according to the Agilent's micro-RNA Microarray System protocol. The arrays were scanned with an Agilent microarray scanner using high dynamic range settings as specified by the manufacturer. Agilent Feature Extraction Software Version 10.7.3.1 was used to extract the data. Normalization was performed on the total Gene Signal from Agilent "GeneView" data files in R language, version 3.4, an open source statistical scripting language (http://www.r-project. org). Invariant-based normalization was performed as previously described ${ }^{55}$. After normalization, micro-RNA not detected in any sample were filtered, leaving 240 miRNAs for analysis. For statistical analyses, we performed linear model using Bioconductor package Limma with the two conditions (chow diet and high-fat diet) as factors, extraction of contrasts of interest, moderated $t$ test for comparisons were applied, adjustment of $P$ values by the Benjamini-Hochberg method and controlling for false discovery rate were realized.

\section{Pathway analysis}

We performed computational target prediction analysis coupled to pathway analysis using DIANA-miRPath (v3.0) software. Potential target RNAs for down-regulated micro-RNAs from each cluster were identified using the prediction algorithm DIANA-microT-CDS (v5.0). The potential mRNA targets for the down-regulated microRNAs were then subjected to KEGG pathway analysis. The DIANA-miRPath software calculates the significance for all the miRNA-mRNA pairs in a pathway, and then combines them into a merged $P$ value for each pathway ${ }^{21}$. 


\section{Real-time quantitative PCR for mRNA expression}

In total, $200 \mathrm{ng}$ of RNAs was reverse transcribed using High Capacity Reverse Transcription Kit according to the manufacturer's protocols in a SimplyAmp Thermal Cycler (Life Technologies, Inc.). RT-qPCR was performed with $2 \mu \mathrm{l}$ of diluted cDNA (1:20) using the I-Taq Universal SybrGreen Master Mix and both sense and antisense primers in a final volume of $10 \mu \mathrm{l}$ in a Quantstudio 3 apparatus (Life Technologies, Inc.). The primers that were used are listed in supplemental table S1. Data were normalized to $\beta$-actin or to Gapdh using the $\mathrm{Ct}$ method.

\section{Micro-RNA expression quantification by RT-qPCR}

In total, $10 \mathrm{ng}$ of RNAs was reverse transcribed with advanced miRNA RT kit on a SimplyAmp Thermal Cycler (Life Technologies, Inc.). RT product was diluted by 10fold, and $2.5 \mu \mathrm{l}$ was used for qPCR analyses. qPCR was performed with TaqMan Advanced Master Mix according to the manufacturer's protocol on a Quantstudio 3 apparatus (Life Technologies, Inc.). The relative expression levels of miRNAs were calculated using the comparative $\Delta \Delta C$ t method by normalizing to miR-16 levels.

\section{Data analysis}

The data from the different experiments were analyzed with GraphPad Prism software version 6.05 (GraphPad Software, Inc.). The values were expressed as mean \pm SEM to account for sample and animal variation within a dataset. Student's $t$-test was performed to determine whether there were differences between two groups, and analysis of variance when multiple groups were compared. $P<0.05$ was considered statistically significant.

\section{Acknowledgements}

The authors acknowledge the C3M animal room facility (Nice), Dr. Sylvain Pradervand, the Genomics Technologies Facility (Lausanne), the mouse pathology facility (Lausanne), and Dr. Fabienne Maurer. This work was financially supported by the CHUV (Centre Hospitalier Universitaire Vaudoix) and the INSERM U1065-Nice-Sophia-Antipolis University.

\section{Authors' contributions}

Conceptualization: S.B., M.B. and U.S.; methodology: S.B., C.M. and H.C.; investigation: S.B., M.L., G.B. and M.C.; format analysis: S.B., C.M., H.C., M.B. and U. S.; resources: M.B. and U.S.; writing: S.B., C.M., H.C., M.B. and U.S.; supervision: S.B., M.B. and U.S.

\section{Conflict of interest}

The authors declare that they have no conflict of interest.

\section{Publisher's note \\ Springer Nature remains neutral with regard to jurisdictional claims in published maps and institutional affiliations.}

The online version of this article (https://doi.org/10.1038/s41420-019-0153-y) contains supplementary material, which is available to authorized users.

Received: 3 January 2019 Revised: 8 February 2019 Accepted: 13 February 2019
Published online: 01 March 2019

\section{References}

1. Jakicic, J. M., Rogers, R. J., Davis, K. K. \& Collins, K. A. Role of physical activity and exercise in treating patients with overweight and obesity. Clin. Chem. 64, 99-107 (2018).

2. Savarese, G. \& Lund, L. H. Global public health burden of heart failure. Card. Fail. Rev. 3, 7-11 (2017).

3. Reynolds, R. M. et al. Maternal obesity during pregnancy and premature mortality from cardiovascular event in adult offspring: follow-up of 1323275 person years. BMJ 347, f4539 (2013).

4. Siddeek, B. et al. Transient postnatal over nutrition induces long-term alterations in cardiac NLRP3-inflammasome pathway. Nutr. Metab. Cardiovasc. Dis. 28, 944-951 (2018).

5. Blackmore, H. L. et al. Maternal diet-induced obesity programs cardiovascular dysfunction in adult male mouse offspring independent of current body weight. Endocrinology 155, 3970-3980 (2014).

6. Maloyan, A. et al. Identification and comparative analyses of myocardial miRNAs involved in the fetal response to maternal obesity. Physiol. Genom. 45, 889-900 (2013).

7. Velkoska, E., Cole, T. J., Dean, R. G., Burrell, L. M. \& Morris, M. J. Early undernutrition leads to long-lasting reductions in body weight and adiposity whereas increased intake increases cardiac fibrosis in male rats. J. Nutr. 138, 1622-1627 (2008)

8. Siddeek, B., Mauduit, C., Yzydorczyk, C., Benahmed, M. \& Simeoni, U. At the heart of programming: the role of micro-RNAs. J. Dev. Orig. Health Dis. 9, 615-631 (2018).

9. Friedman, R. C., Farh, K. K., Burge, C. B. \& Bartel, D. P. Most mammalian mRNAs are conserved targets of microRNAs. Genome Res. 19, 92-105 (2009).

10. Chen, J. F. et al. Targeted deletion of Dicer in the heart leads to dilated cardiomyopathy and heart failure. Proc. Natl. Acad. Sci. USA 105, 2111-2116 (2008).

11. da Costa Martins, P. A. et al. Conditional dicer gene deletion in the postnatal myocardium provokes spontaneous cardiac remodeling. Circulation 118, 1567-1576 (2008).

12. Rao, P. K. et al. Loss of cardiac microRNA-mediated regulation leads to dilated cardiomyopathy and heart failure. Circ. Res. 105, 585-594 (2009).

13. Han, J. et al. The Drosha-DGCR8 complex in primary microRNA processing. Genes Dev. 18, 3016-3027 (2004)

14. Yi, R., Qin, Y., Macara, I. G. \& Cullen, B. R. Exportin-5 mediates the nuclear export of pre-microRNAs and short hairpin RNAs. Genes Dev. 17, 3011-3016 (2003).

15. Wilson, R. C. et al. Dicer-TRBP complex formation ensures accurate mammalian microRNA biogenesis. Mol. Cell 57, 397-407 (2015).

16. Maniataki, E. \& Mourelatos, Z. A human, ATP-independent, RISC assembly machine fueled by pre-miRNA. Genes Dev. 19, 2979-2990 (2005).

17. Davis, C. D. \& Ross, S. A. Evidence for dietary regulation of microRNA expression in cancer cells. Nutr. Rev. 66, 477-482 (2008).

18. Fiedler, S. D., Carletti, M. Z., Hong, X. \& Christenson, L. K. Hormonal regulation of MicroRNA expression in periovulatory mouse mural granulosa cells. Biol. Reprod. 79, 1030-1037 (2008).

19. Kulshreshtha, R. et al. Regulation of microRNA expression: the hypoxic component. Cell Cycle 6, 1426-1431 (2007).

20. Hudder, A. \& Novak, R. F. miRNAs: effectors of environmental influences on gene expression and disease. Toxicol. Sci. 103, 228-240 (2008).

21. Vlachos, I. S. et al. DIANA miRPath v.2.0: investigating the combinatorial effect of microRNAs in pathways. Nucleic Acids Res. 40, W498-W504 (2012).

22. Dobaczewski, M., Chen, W. \& Frangogiannis, N. G. Transforming growth factor (TGF)-beta signaling in cardiac remodeling. J. Mol. Cell. Cardiol. 51, 600-606 (2011).

23. Faller, M., Matsunaga, M., Yin, S., Loo, J. A. \& Guo, F. Heme is involved in microRNA processing. Nat. Struct. Mol. Biol. 14, 23-29 (2007).

24. Barr, I. et al. DiGeorge critical region 8 (DGCR8) is a double-cysteine-ligated heme protein. J. Biol. Chem. 286, 16716-16725 (2011).

25. Barr, l. et al. Ferric, not ferrous, heme activates RNA-binding protein DGCR8 for primary microRNA processing. Proc. Natl. Acad. Sci. USA 109, 1919-1924 (2012).

26. Weitz, S. H., Gong, M., Barr, I., Weiss, S. \& Guo, F. Processing of microRNA primary transcripts requires heme in mammalian cells. Proc. Natl. Acad. Sci. USA 111, 1861-1866 (2014). 
27. Worou, M. E. et al. Hemin decreases cardiac oxidative stress and fibrosis in a rat model of systemic hypertension via PI3KJAkt signalling. Cardiovasc. Res. 91, 320-329 (2011).

28. Zhu, X. et al. HMOX-1 inhibits TGF-beta-induced epithelial-mesenchymal transition in the MCF-7 breast cancer cell line. Int. J. Mol. Med. 40, 411-417 (2017).

29. De Jong, K. A. et al. Maternal high fat diet induces early cardiac hypertrophy and alters cardiac metabolism in Sprague Dawley rat offspring. Nutr. Metab. Cardiovasc. Dis. 28, 600-609 (2018).

30. Bilandzic, M. \& Stenvers, K. L. Betaglycan: a multifunctional accessory. Mol. Cell. Endocrinol. 339, 180-189 (2011).

31. Lopez-Casillas, F., Wrana, J. L. \& Massague, J. Betaglycan presents ligand to the TGF beta signaling receptor. Cell 73, 1435-1444 (1993).

32. Groeneveld, M. E. et al. Betaglycan (TGFBR3) up-regulation correlates with increased TGF-beta signaling in Marfan patient fibroblasts in vitro. Cardiovasc. Pathol. 32, 44-49 (2018).

33. Liao, P. et al. The in vivo role of p38 MAP kinases in cardiac remodeling and restrictive cardiomyopathy. Proc. Natl. Acad. Sci. USA 98, 12283-12288 (2001).

34. Karayiorgou, M., Simon, T. J. \& Gogos, J. A. 22q11.2 microdeletions: linking DNA structural variation to brain dysfunction and schizophrenia. Nat. Rev. Neurosci. 11, 402-416 (2010).

35. Shiohama, A., Sasaki, T., Noda, S., Minoshima, S. \& Shimizu, N. Molecular cloning and expression analysis of a novel gene DGCR8 located in the DiGeorge syndrome chromosomal region. Biochem. Biophys. Res. Commun. 304, 184-190 (2003)

36. Stark, K. L. et al. Altered brain microRNA biogenesis contributes to phenotypic deficits in a 22q11-deletion mouse model. Nat. Genet. 40, 751-760 (2008).

37. Marino, B., Digilio, M. C., Toscano, A., Giannotti, A. \& Dallapiccola, B. Congenital heart defects in patients with DiGeorge/velocardiofacial syndrome and del22q11. Genet. Couns. 10, 25-33 (1999).

38. Noble, P. W. et al. Pirfenidone in patients with idiopathic pulmonary fibrosis (CAPACITY): two randomised trials. Lancet 377, 1760-1769 (2011).

39. Gallo, E. M. et al. Angiotensin I-dependent TGF-beta signaling contributes to Loeys-Dietz syndrome vascular pathogenesis. J. Clin. Invest. 124, 448-460 (2014).

40. Habashi, J. P. et al. Losartan, an AT1 antagonist, prevents aortic aneurysm in a mouse model of Marfan syndrome. Science 312, 117-121 (2006).
41. Gong, M. et al. Caspases cleave and inhibit the microRNA processing protein DiGeorge Critical Region 8. Protein Sci. 21, 797-808 (2012).

42. Herbert, K. M., Pimienta, G., DeGregorio, S. J., Alexandrov, A. \& Steitz, J. A. Phosphorylation of DGCR8 increases its intracellular stability and induces a progrowth miRNA profile. Cell Rep. 5, 1070-1081 (2013).

43. Wada, T., Kikuchi, J. \& Furukawa, Y. Histone deacetylase 1 enhances microRNA processing via deacetylation of DGCR8. EMBO Rep. 13, 142-149 (2012).

44. Nguyen, T. A., Park, J., Dang, T. L., Choi, Y. G. \& Kim, V. N. Microprocessor depends on hemin to recognize the apical loop of primary microRNA. Nucleic Acids Res. 46, 5726-5736 (2018).

45. Barr, I. et al. Cobalt(III) protoporphyrin activates the DGCR8 protein and can compensate microRNA processing deficiency. Chem. Biol. 22, 793-802 (2015).

46. Izzotti, A. et al. Downregulation of microRNA expression in the lungs of rats exposed to cigarette smoke. FASEB J. 23, 806-812 (2009).

47. Abdelmohsen, K., Srikantan, S., Kang, M. J. \& Gorospe, M. Regulation of senescence by microRNA biogenesis factors. Ageing Res. Rev. 11, 491-500 (2012).

48. Williams, M., Cheng, Y. Y., Blenkiron, C. \& Reid, G. Exploring mechanisms of microRNA downregulation in cancer. Microrna 6, 2-16 (2017).

49. Beveridge, N. J., Gardiner, E., Carroll, A. P., Tooney, P. A. \& Cairns, M. J. Schizophrenia is associated with an increase in cortical microRNA biogenesis. Mol. Psychiatry 15, 1176-1189 (2010).

50. Bartram, M. P. et al. Loss of Dgcr8-mediated microRNA expression in the kidney results in hydronephrosis and renal malformation. BMC Nephrol. 16, 55 (2015).

51. Jeker, L. T, Zhou, X, Blelloch, R. \& Bluestone, J. A. DGCR8-mediated production of canonical microRNAs is critical for regulatory $T$ cell function and stability. PLOS ONE 8, e66282 (2013).

52. Siddeek, B. et al. Developmental epigenetic programming of adult germ cell death disease: polycomb protein EZH2-miR-101 pathway. Epigenomics 8, 1459-1479 (2016).

53. Siddeek, B. et al. MicroRNAs as potential biomarkers in diseases and toxicology. Mutat. Res Genet Toxicol. Environ. Mutagen 764-765, 46-57 (2014).

54. Chen, Y., Yu, Q. \& Xu, C.-B. A convenient method for quantifying collagen fibers in atherosclerotic lesions by ImageJ software. Int. J. Clin. Exp. Med. 10, 14904-14910 (2017).

55. Pradervand, $\mathrm{S}$. et al. Impact of normalization on miRNA microarray expression profiling. RNA 15, 493-501 (2009). 